\title{
A monoclonal natural human lgM protects axons in the absence of remyelination
}

\author{
Bharath Wootla ${ }^{1,2,3+}$, Aleksandar Denic ${ }^{1,2+}$, Arthur E. Warrington ${ }^{1,2}$ and Moses Rodriguez ${ }^{1,2,4^{*}}$
}

\begin{abstract}
Background: Whereas demyelination underlies early neurological symptoms in multiple sclerosis (MS), axonal damage is considered critical for permanent chronic deficits. Intracerebral infection of susceptible mouse strains with Theiler's murine encephalomyelitis virus (TMEV) results in chronic induced demyelinating disease (TMEV-IDD) with progressive axonal loss and neurologic dysfunction similar to progressive forms of MS. We previously reported that treatment of chronic TMEV-IDD mice with a neurite outgrowth-promoting natural human antibody, HIgM12, improved brainstem NAA concentrations and preserved functional motor activity. In order to translate this antibody toward clinical trial, we generated a fully human recombinant form of HlgM12, rHIgM12, determined the optimal in vivo dose for functional improvement in TMEV-IDD, and evaluated the functional preservation of descending spinal cord axons by retrograde labeling.

Findings: SJL/J mice at 45 to 90 days post infection (dpi) were studied. A single intraperitoneal dose of $0.25 \mathrm{mg} / \mathrm{kg}$ of $\mathrm{rHlgM} 12$ per mouse is sufficient to preserve motor function in TMEV-IDD. The optimal dose was $10 \mathrm{mg} / \mathrm{kg}$. rHIgM12 treatment protected the functional transport in spinal cord axons and led to $40 \%$ more Fluoro-Gold-labeled brainstem neurons in retrograde transport studies. This suggests that axons are not only present but also functionally competent. rHlgM12-treated mice also contained more mid-thoracic (T6) spinal cord axons than controls.

Conclusions: This study confirms that a fully human recombinant neurite outgrowth-promoting monoclonal $\mathrm{IgM}$ is therapeutic in a model of progressive MS using multiple reparative readouts. The minimum effective dose is similar to that of a remyelination-promoting monoclonal human IgM discovered by our group that is presently in clinical trials for MS.
\end{abstract}

Keywords: Multiple sclerosis, Theiler's murine encephalomyelitis virus, Brainstem, Axons, Retrograde labeling, Activity monitoring

\section{Findings}

\section{Introduction}

Multiple sclerosis (MS) is an inflammatory demyelinating disease of the central nervous system (CNS). Axonal damage is considered critical for permanent chronic deficits in progressive MS, but the precise mechanisms by which axonal injury occurs in MS are unclear. None of the currently FDA-approved drugs or candidates in clinical trial protect neurons and axons from degeneration. Theiler's murine encephalomyelitis virus (TMEV) is a single-

\footnotetext{
* Correspondence: Rodriguez.Moses@mayo.edu

${ }^{\dagger}$ Equal contributors

'Department of Neurology, Mayo Clinic College of Medicine, Mayo Clinic, 200 First Street SW, Rochester, MN 55905, USA

${ }^{2}$ Mayo Clinic Center for Multiple Sclerosis and Autoimmune Neurology, Mayo

Clinic, 200 First Street SW, Rochester, MN 55905, USA

Full list of author information is available at the end of the article
}

stranded RNA virus that belongs to the Picornaviridae family. Intracerebral injection of TMEV induces an inflammatory demyelinating disease in the spinal cord of susceptible strains of mice following infection [1]. TMEVinduced demyelinating disease (TMEV-IDD) is a natural chronic progressive CNS demyelinating disease of susceptible strains of mice, with similarities to primary progressive MS [2]. The TMEV-induced model of demyelinating disease is a reasonable platform for therapeutic drug discovery for progressive forms of demyelination.

We previously reported that a human IgM monoclonal antibody, HIgM12, bound to the surface of neurons, supported robust neurite extension when presented as a substrate, and overrode the neurite extension inhibition of CNS myelin and myelin-associated glycoprotein (MAG) [3-5]. We also showed that a single $10 \mathrm{mg} / \mathrm{kg}$ 
dose of HIgM12, when peripherally administered, improved clinical disease course of chronic virus-infected mice beginning 2 weeks following treatment, which persisted for 8 weeks [6]. In a recent follow-up study, HIgM12, when administered intraperitoneally to 90-day TMEV-IDD mice, improved brainstem NAA concentrations, a surrogate biomarker for the density of spinal cord axons, at 5 and 10 weeks following treatment [7]. This improvement was present even though HIgM12 does not promote spinal cord remyelination. A reagent that protects axons without associated remyelination would be a critical drug by itself or paired with immunemodulatory or remyelination-promoting therapies. To translate this IgM to clinical trial, we generated a fully human recombinant form of the IgM (rHIgM12) from $\mathrm{CHO}$ cells and tested this for efficacy in the TMEV model. We first determined the minimum and optimum dose of rHIgM12 that improves neurologic function in vivo by locomotor monitoring of TMEV-IDD mice.

Active axonal retrograde labeling relies on both anatomically continuous axons and preserved retrograde transport mechanism which provides an assessment of axonal integrity. Retrograde labeling analysis of spinal cord axons in chronically demyelinated mice previously revealed a marked reduction in the number of labeled neuronal cell bodies in the brainstem [8]. Our recent report [7] describing an increase in brainstem NAA concentrations after treatment with HIgM12 led us to determine whether treatment with rHIgM12 results in an increased number of retrograde tracer-positive neuron cell bodies within the brainstem, indicating functional transport.

\section{Methods}

\section{Ethics statements}

The Mayo Clinic Institutional Animal Care and Use Committee (IACUC) approved all animal protocols used in this study.

\section{Theiler's virus model of demyelination}

Demyelinating disease was induced in 8-week-old SJL/J mice by intracerebral injection of $10 \mu \mathrm{l}$ containing $2.0 \times 10^{5}$ plaque-forming units (PFUs) of Daniel's strain TMEV [9]. This typically results in $>98 \%$ incidence of infection with rare fatalities.

\section{Antibodies}

Human IgM12 was identified and isolated from a patient $\left(\mathrm{N}^{\circ} 12\right)$ with Waldenstrom's macroglobulinemia carrying high levels of this monoclonal protein for years without detriment. The variable region amino acid and nucleotide sequences for the heavy and light chains are available in GenBank under the accession numbers DQ146928 and DQ146929, respectively. rHIgM12 was expressed in CHO-
S cells (GibcoBRL, cat\# 11619). Plasmids expressing the heavy and light chain coding sequences were cotransfected along with a human J chain. The resulting cells were selected with increasing doses of methotrexate, and clones that produced IgM as measured by ELISA were sub-cloned and expanded. A stable clone was expanded, banked, and successfully grown in hollow fiber or wave bags to obtain supernatant. rHIgM12 was purified in a qualified GLP facility. Briefly, diafiltered culture supernatant containing rHIgM12 was subjected to a three-step purification protocol (Fig. 1a). First, culture supernatant was loaded on a CHT Ceramic Hydroxyapatite, Type II (Bio-Rad) column and eluted using $250 \mathrm{mM}$ sodium phosphate $(\mathrm{NaOH}, \mathrm{pH}$ 7.0). The eluted solution was then passed through a CIM QA-8f Monolithic column (BIA Separations) followed by elution with $250 \mathrm{mM} \mathrm{NaOH}$, $500 \mathrm{mM} \mathrm{NaCl}(\mathrm{pH}$ 7.0). In the final step of purification, the eluate from the second step was further subjected to separation on a Sephacryl S-300 HR column in the presence of $50 \mathrm{mM} \mathrm{Na}_{2} \mathrm{HPO}_{4}, 150 \mathrm{mM} \mathrm{NaCl}$ (pH 7.0). Collection of only peak fractions at the final step allowed approximately 6-fold concentration of the eluate from the monolith column. The purity of eluates at each step is shown (Fig. 1b, c). The stock rHIgM12 preparation was stored as $5 \mathrm{mg} / \mathrm{ml}$ aliquots. The final IgM was validated using titrated potency assays of binding to the surface of neurons and support of neurite extension. The potency of rHIgM12 and the serum-derived IgM were compared [10] and found to be identical therapeutically. rHIgM12 can be obtained for non-commercial experimental use by contacting the corresponding author. The control human IgM used was isolated from human serum [4]. It did not bind to neurons in culture or live tissue slices nor support neurite extension in cell culture. Informed consent was obtained from all patients for the use of serum samples for research purposes.

\section{Treatment of mice}

Dosing study: SJL mice at 45 days post infection (dpi), the time point when demyelination in the spinal cord is well established and before substantial axon loss, were treated with a single intraperitoneal dose of rHIgM12 $(0.25,2.5$, 10 , or $25 \mathrm{mg} / \mathrm{kg}$ ) or isotype control IgM $(10 \mathrm{mg} / \mathrm{kg})$. Dosing was based on the average weight of an adult SJL mouse $(20 \mathrm{~g})$. Retrograde labeling study: SJL mice at 90 dpi, the time point when axonal degeneration is present and progressing, were treated with a single intraperitoneal dose of rHIgM12 $(10 \mathrm{mg} / \mathrm{kg})$ or saline.

\section{Spontaneous activity monitoring}

Spontaneous horizontal and vertical locomotor activity was recorded using open-field (OF) locomotor activity monitors (Omnitech Electronics; Columbus, $\mathrm{OH}$ ) and Fusion v5.0 software. Each monitor consists of sets of 16 


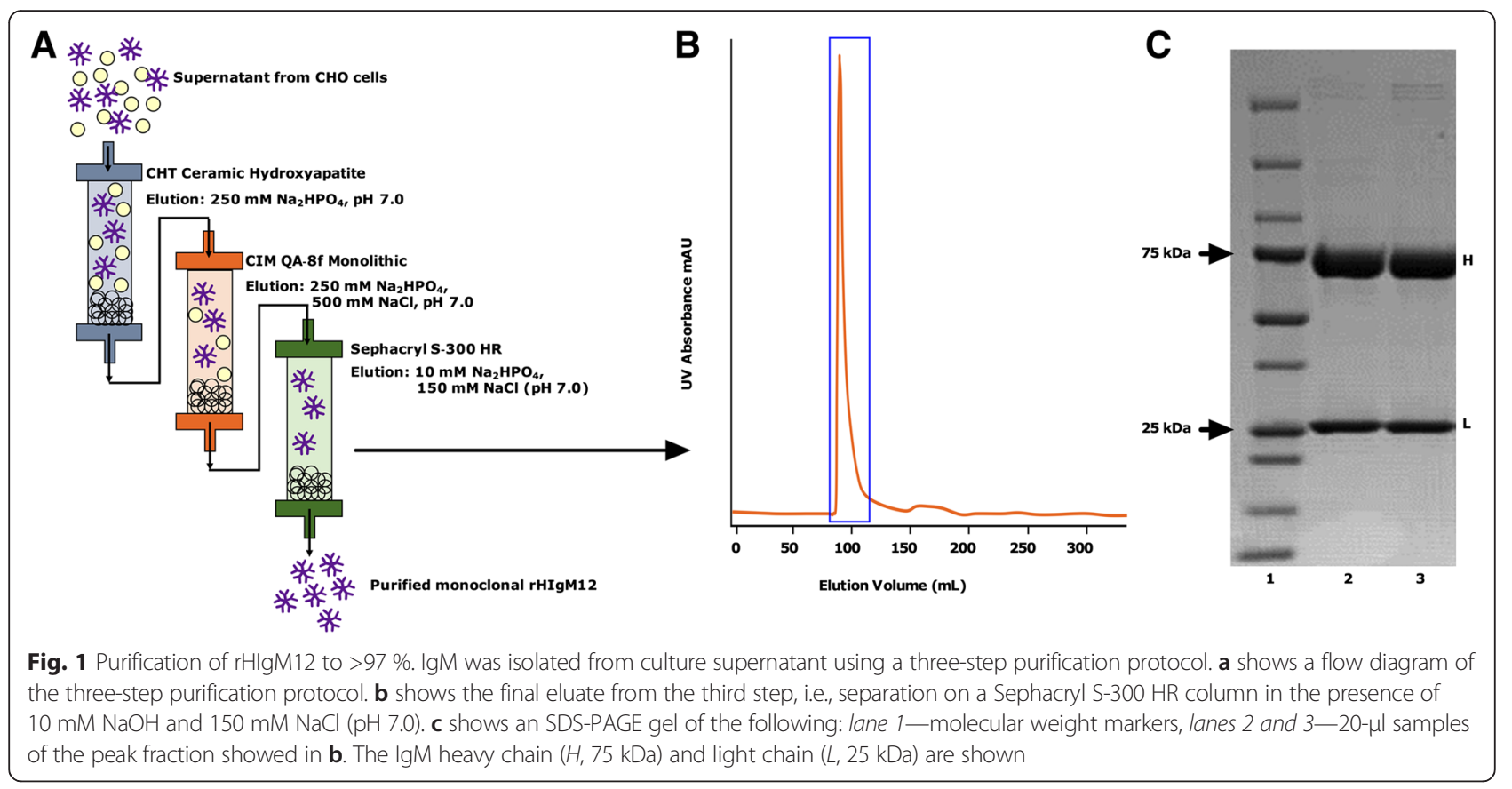

light beam arrays in the horizontal $x$ and $y$ axes. The hardware detects beams broken by animal movements to determine the location within the cage. In all cages, mice were exposed to identical environmental conditions: (a) freely accessible food and water; (b) a normal 12-h light/dark cycle; and (c) $70{ }^{\circ} \mathrm{F}$ ambient temperature. Five SJL mice at $45 \mathrm{dpi}$ were placed in each cage, and baseline spontaneous activity was collected over a period of 5 consecutive days. Groups of mice were then treated with a single dose of rHIgM12 $(0.25,2.5,10$, or $25 \mathrm{mg} / \mathrm{kg})$ or with $10 \mathrm{mg} / \mathrm{kg}$ of control human IgM antibody. Following treatment, mice were continuously monitored for 56 days. The total horizontal and vertical activity data, quantified as mean hourly mean breaks, was exported to an Excel (Microsoft Corporation) compatible file for further analysis. The original activity box data sets were first normalized to baseline activity independently for each group of mice (Fig. 2a, b) followed by a polynomial curve fitting (Fig. 2c, d). We described this methodology in greater detail (see [6]). Briefly, the model was designed to allow for polynomial terms up to any degree (xn) and estimated shape parameters separately for each dose and treatment group. For the analysis of datasets in this study, we chose the third-degree polynomial followed by normalization of curves to $y$ axis $=0$. This allowed us sufficient flexibility to model non-linear effects over time. Polynomial analysis of data sets was considered ideal as it allowed us to perform direct pairwise comparison at regular intervals across the entire time frame to determine when treatment groups have significantly diverged. In addition, a polynomial fit clarifies data by eliminating noise to draw visual attention on the general trend over the entire time frame. Polynomial regression models were performed and plotted using Graphpad Prism v5.0.

\section{Retrograde labeling}

Retrograde labeling [8] of brainstem neurons was performed on 23 mice (uninfected $=3, \operatorname{rHIgM} 12=9$, saline $=11$ ) at 9 weeks after antibody treatment. Briefly, dorsal laminectomy of the lower thoracic vertebrae was performed on anesthetized mice. The spinal cord was hemisected on the right side using microdissecting blades. The retrograde tracer, Fluoro-Gold, was applied and the surgery site was closed. One week post-surgery (i.e., 10 weeks post-treatment), mice were terminally anesthetized, perfused with $4 \%$ paraformaldehyde, and brains and spinal cords were collected. Serial vibratome (Lancer Series 1200) sections ( $40 \mu \mathrm{m}$ thick) of the brainstem were collected and mounted under Vectashield (Vector Laboratories Inc., Burlingame, CA). Cell bodies containing retrograde tracer (Fluoro-Gold), visualized by UV illumination (360-370-nm excitation, 420-460-nm emission) were counted at $200 \times$ from 16 brainstem slices per mouse. A cell was counted as positive if a large cross-section of the cell body was labeled with FluoroGold. All analyses were performed without knowledge of the experimental groups.

\section{Spinal cord morphometry}

We assessed spinal cord morphometry in all groups 10 weeks following treatment. Mice were anesthetized with sodium pentobarbital and perfused intracardially with Trump's fixative (phosphate-buffered $4 \%$ formaldehyde/ 


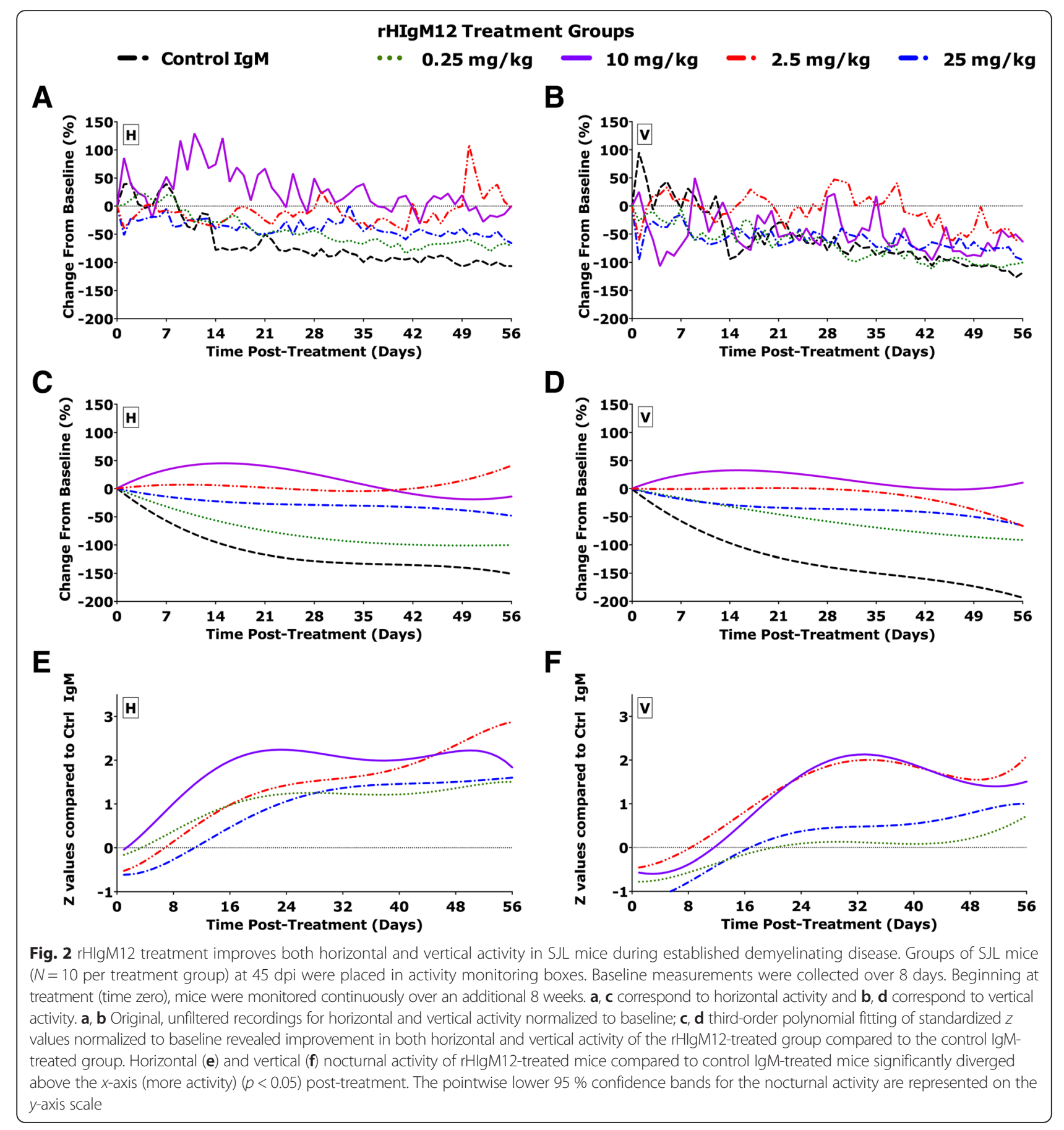

$1 \%$ glutaraldehyde, $\mathrm{pH}$ 7.4). Spinal cords were removed and sectioned precisely into 1 -mm blocks. In order to represent samples along the length of the spinal cord, every third block was postfixed, stained with osmium tetroxide, and embedded in araldite plastic (Polysciences, Warrington, PA). One-micrometer sections were cut and stained with $4 \%$ p-phenylenediamine to visualize the myelin sheaths. We examined ten spinal cord cross-sections, spanning the entire spinal cord from cervical to the distal lumbar regions, from each mouse. Each spinal cord quadrant from every coronal section was graded for the presence of inflammation and demyelination. Quadrants with inflammation were defined by the presence of inflammatory cells lining the meninges. Areas of demyelination were defined as containing naked axons, macrophage infiltration, myelin ovoids, and degenerated axon profiles. These areas were well demarcated and allow accurate quantitative assessment at $10 \times$ and $40 \times$ magnifications, respectively. Demyelination 
or inflammation scores were expressed as the percentage of spinal cord quadrants examined with pathological abnormality. A maximum score of 100 indicated pathological abnormality in every quadrant of all spinal cord sections of a given mouse. All grading was performed on coded sections without knowledge of the experimental group.

To quantify myelinated axons, a mid-thoracic (T6) spinal cord section from each animal was examined. This level of the cord was chosen because it contains both ascending axons and descending axons some of which have neuronal bodies in the brainstem [11]. To ensure a uniform intensity of myelin labeling, all spinal cord T6 sections used in the study were stained with the same batch of $4 \%$ para-phenylenediamine for exactly 20 min. An Olympus Provis AX70 microscope and a $60 \times$ oil-immersion objective were used to capture six sample areas of normal-appearing white matter without demyelination from each section. The fields were collected in a clockwise manner around the section to obtain representative samplings of the posterior-lateral, antero-lateral, and anterior columns. Images were centered between the gray matter and meningeal surface. Approximately $400,000 \mu \mathrm{m}^{2}$ of white matter was sampled from each mouse. Absolute myelinated axon numbers were measured using automated counting software that recognizes circular intact myelin sheathes and calculated as reported [12]. Data were represented as the absolute number of all axons sampled per mid-thoracic spinal cord section. All values were averaged per group.

\section{Statistics}

Data from activity monitoring: Statistical comparisons of treatment groups were performed using the predicted model values and respective standard errors based on the $z$-statistic (SAS Institute, Inc.). Direct pairwise comparisons of treatments were performed for each day across the entire time frame, and statistical significance was determined at the typical $a=0.05$ threshold. No adjustments were made for multiple comparisons. Data for retrograde labeling and axon-count analysis were compared by Student's $t$ test if normally distributed or by Mann-Whitney rank sum test if non-normally distributed. In all analyses, $p<0.05$ was considered as statistically significant. Correlation coefficients between paired sets of data were determined using the Pearson product moment correlation.

\section{Results}

Nocturnal behavior is a sensitive measure of neurologic deficits in TMEV-IDD [6]. In this study, we used this assay to determine the minimum and the most effective dose of rHIgM12 that improves spontaneous horizontal and vertical locomotor activity in TMEV-IDD. To address this, we monitored 45 dpi TMEV-IDD mice ( $N=10$ two groups of
5 mice per treatment) treated with either rHIgM12 at 0.25 , $2.5,10$, or $25 \mathrm{mg} / \mathrm{kg}$ or control human IgM $(10 \mathrm{mg} / \mathrm{kg})$. Following treatment, spontaneous activity was recorded continuously over 8 weeks. The original raw activity data is noisy (Fig. 2a, b). In order to allow a better visual comparison, we subjected the data to third-degree polynomial curve fitting of standardized $z$ values (Fig. 2c, d). This allowed a visual comparison of groups. Using direct pairwise comparisons (Fig. 2e, f) of activity after polynomial fitting, we determined that improved horizontal nocturnal motor function in rHIgM12-treated mice became statistically significant at days $6,9,3$, and 14 post-treatment for the 0.25-, 2.5-, 10-, and $25-\mathrm{mg} / \mathrm{kg}$ doses, respectively, as compared to control IgM (Fig. 2e). Improvement in horizontal nocturnal activity of rHIgM12-treated animals persisted until the end of experiment at 8 weeks. Improved vertical nocturnal motor function in rHIgM12-treated mice became statistically significant at days 12, 15, and 23 post-treatment for the 2.5-, $10-$, and $25-\mathrm{mg} / \mathrm{kg}$ doses, respectively, as compared to control IgM (Fig. 2f). Vertical activity in the $0.25-\mathrm{mg} / \mathrm{kg}$ dose group was not statistically significant at any time point post-treatment when compared to control IgM.

We recently reported that treatment of TMEVinfected SJL mice with the myelin/oligodendrocyte-reactive human IgM, rHIgM22, resulted in more retrogradely labeled neuronal cell bodies in the brainstem indicating that improving the level of remyelination can preserve function in spinal cord axons [13]. We used the same retrograde labeling assay to investigate whether treatment with rHIgM12, which does not improve the levels of remyelination, could directly protect neurons in the brain stem and spinal cord axons. Functional preservation of spinal cord axons may underlie rHIgM12 improvement of brainstem NAA concentrations [7] and locomotor activity. Retrograde labeling relies on both anatomically continuous axons and preserved retrograde transport mechanisms. We established TMEV-IDD in 20 susceptible SJL mice. Nine mice were treated at $90 \mathrm{dpi}$ with $10 \mathrm{mg} / \mathrm{kg}$ of $\mathrm{rHIgM} 12$; the remaining 11 mice were administered through vehicle. At 9 weeks posttreatment, we performed retrograde labeling on all 23 mice ( uninfected $=3$, rHIgM12 $=9$, saline $=11$ ). Figure 3a shows an example of a cluster of fluorescently labeled neurons in the brainstem, where cell bodies as well as dendrites and axons are clearly seen. For each descending neuron population, cell bodies containing retrogradely transported Fluoro-Gold label were quantified. Uninfected mice $(N=3)$ had an average number of $2983 \pm 39$ (mean \pm SEM) labeled brainstem neurons. Retrograde labeling studies performed in demyelinated mice demonstrated a large reduction in fluorescently labeled neuron cell bodies in the brainstem [8]. When we quantified fluorescently labeled brainstem neurons in TMEV-IDD mice, as expected, we found fewer labeled neurons compared to uninfected mice. 

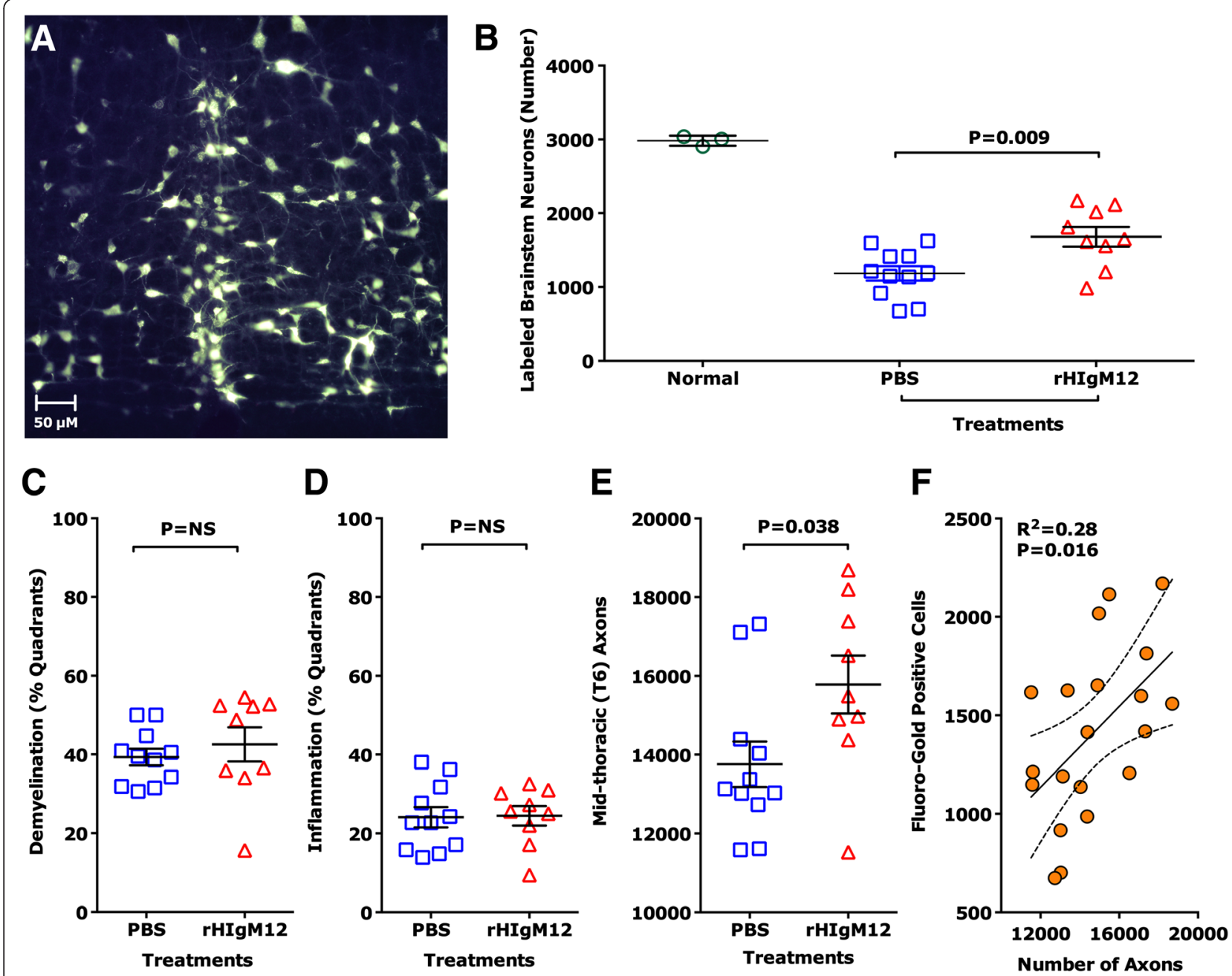

Fig. 3 rHlgM12 treatment improves the number of retrograde-labeled brainstem neurons and preserves spinal cord axons, but does not affect spinal cord demyelination. a Fluoro-Gold-labeled neurons were counted in brain stem sections. The panel shows an example of a cluster of fluorescently labeled neurons in the brainstem. Extensive labeling of cell bodies as well as axons and dendrites can be easily appreciated. $\mathbf{b} \mathrm{rHIgM12}$ treatment increased the number of retrograde-labeled brainstem neurons compared to the saline-treated group $(p=0.009$, Mann-Whitney rank sum test). The number of labeled neurons in uninfected positive control mice $(N=3$, circles) is shown for reference. Forest plots show the average number of retrograde-labeled brainstem neurons \pm SEM per treatment group: rHIgM12 (red triangles) and saline (blue boxes). Mice from both treatment groups had similar levels of spinal cord $\mathbf{c}$ demyelination and $\mathbf{d}$ inflammation pathology. Pathology analysis was performed blinded. $\mathbf{e}$ When the number of myelinated mid-thoracic-level spinal cord axons was compared between treatment groups, rHIgM12-treated mice contained $14.7 \%$ more axons than the saline-treated group ( $p=0.038$, Mann-Whitney rank sum test). $\mathbf{f}$ The number of fluorescent retrograde-labeled brain stem neurons in each mouse correlated positively and significantly with the number of thoracic-level myelinated spinal cord axons $\left(p=0.016, R^{2}=0.28\right)$

We counted on average $1185 \pm 98$ labeled neurons in the control-treated animals $(N=11)$ and $1682 \pm 134$ labeled neurons in rHIgM12-treated animals $(N=9)$. The difference in labeled neuron numbers between the rHIgM12treated group compared to the saline-treated group was significantly different ( $p=0.009$, Mann-Whitney test, twotailed) (Fig. 3b).

We investigated the spinal cord pathology at 10 weeks post-treatment. Ten plastic-embedded cross-sections encompassing the entire length of the cord from each mouse were scored for demyelination (Fig. 3c) and inflammation (Fig. 3d). Pathological scores were similar between both groups of mice. We then determined the number of intact axons present in mid-thoracic (T6) spinal cord sections. Six areas encompassing approximately $400,000 \mu \mathrm{m}^{2}$ of white matter were systematically sampled from each mouse, and the total number of midthoracic axons was compared across treatment groups (Fig. 3e). We found more axons in the rHIgM12-treated group compared to the saline-treated group $(15,782 \pm$ 736 vs $13,758 \pm 575, p=0.038$ ). When the quantity of retrogradely labeled brainstem neurons was plotted as a function of the number of intact myelinated axons at the T6 level from all animals, irrespective of the treatment 
groups (Fig. 3f), we found a positive and significant correlation $\left(R^{2}=0.28 ; p=0.016\right)$.

\section{Discussion}

There is much evidence to support the goal of remyelination as a means to prevent axon degeneration and slow deficit progression in neurologic disease [14]. In the year 2000, we reported the identification of a natural human IgM that promoted robust spinal cord remyelination in both the TMEV-IDD and lysolecithin-induced demyelination models $[15,16]$. A recombinant form of this human IgM, termed rHIgM22, was expressed in a F3B6 cell line with the assembled IgM containing a mouse J chain [17]. rHIgM22 binds to myelin and the surface of oligodendrocytes (OL) and in pre-clinical studies is effective in vivo at very low doses. A single $0.025 \mathrm{mg} / \mathrm{kg}$ intraperitoneal injection of rHIgM22 given to TMEV-IDD mice with demyelination promoted significant remyelination 5 weeks later [18] and increased brainstem NAA concentrations [13], indicating a preservation of axon health [19]. In a recently concluded dose escalation clinical trial in humans with MS, rHIgM22 was tested at doses ranging from 0.025 up to $2 \mathrm{mg} / \mathrm{kg}$ and found to be completely safe [20].

A second human IgM, rHIgM12, that promotes neurite extension [4], improves brainstem NAA concentrations without associated spinal cord remyelination, suggesting that IgM-mediated neuroprotection can be achieved by an alternate mechanism. In the current study, we determined the minimum and optimum dose of rHIgM12 to preserve spontaneous nocturnal function in groups of TMEV-IDD mice. A dose of $0.25 \mathrm{mg} / \mathrm{kg}$ of rHIgM12 improved only horizontal motor function, whereas a dose of $2.5 \mathrm{mg} / \mathrm{kg}$ improved both horizontal and vertical activities. An optimum dose of $10 \mathrm{mg} / \mathrm{kg}$ provided the best improvement of both horizontal and vertical nocturnal motor function and animals treated with a higher dose of $25 \mathrm{mg} / \mathrm{kg}$ did not perform any better. This study suggests that the single dose of rHIgM12 required for biological efficacy in mice is similar to the dose of rHIgM22 required for remyelination. Following identification of the optimum dose, we used the $10 \mathrm{mg} / \mathrm{kg}$ dose of rHIgM12 to obtain direct evaluation of descending axon integrity with retrograde labeling at the T6 level of the spinal cord. Our previous work demonstrated that demyelination is normally accompanied by a reduction of axonal transport $[8,21]$. The majority of demyelinated lesions in TMEV-IDD occur at the cervical and thoracic levels. Therefore, a reduction in the number of labeled brainstem cells occurs primarily because of disturbed retrograde transport or axonal degeneration. In this study, treatment of SJL mice at $90 \mathrm{dpi}$ with a single dose of rHIgM12 protected the function of spinal cord axons; resulting in $42 \%$ more Fluoro-Gold-labeled brainstem neurons (Fig. 3b) and $14.7 \%$ more axons in the rHIgM12 group compared to the control IgM group. This suggests that a preservation of less than $50 \%$ of brainstem neurons and axons was sufficient to observe functional effects, i.e., an improvement in spontaneous locomotor activity. Our previous magnetic resonance spectroscopy study [7] and neuron/axon-count analyses in the current study provide strong evidence that the neurite outgrowth-promoting antibody, rHIgM12, affected neuronal viability through the preservation of axons. The concept that the degree of axonal damage occurs independently of the extent of chronic demyelination is supported by our data and several other studies that examined gray matter lesions and meningeal infiltrates in human brain and genetically manipulated mice [22-24].

The observed preservation of axons by rHIgM12 may relate to the initially described neurite-promoting activity. Studies of several classic neurotrophins link the promotion of neurite outgrowth with neuroprotection $[25,26]$. However, using these pleiotropic molecules in the clinic has been problematic. Antibodies directed against the myelin-derived protein Nogo-A promotes neurite outgrowth in the presence of normally inhibitory molecules [27] and in vivo are therapeutic in models of ALS [28], stroke [29], and spinal cord injury [30]. Treatment of rats with Herceptin, a high-affinity IgG directed against the extracellular domain of the human epidermal growth factor receptor 2 protein (HER2), enhanced axon regeneration after peripheral nerve injury [31]. rHIgM12 binds with high affinity to gangliosides (GD1a and GT1b) [32] and to polysialylated-neural cell adhesion molecule (PSANCAM) [33]. GD1a and GT1b are also ligands for the neurite inhibitory molecule myelin-associated glycoprotein (MAG) and facilitate interactions between OLs and axons that maintain long-term axonal stability [34]. MAG-induced inhibition of axon extension involves the rearrangement of neuronal membrane domains and recruitment of p75 neurotrophin receptor to those domains [35]. rHIgM12 binding to the neuronal surface also rearranges plasma-membrane microdomains and clusters signaling molecules, resulting in a shift of microtubule stability and dynamics [36]. We hypothesize that rHIgM12-mediated neurite outgrowth and protection may use the same membrane platforms targeted by antibodies to the neurite outgrowth inhibitor A (Nogo-A) and the leucine rich repeat and immunoglobulin-like domaincontaining protein 1 (LINGO-1) [37, 38]. Given that rHIgM12 is produced as a fully human antibody, we expect no toxicity even at higher doses in human studies. Among these neuroprotective reagents, only rHIgM12 was isolated from a human. Most antibody-based therapeutics are humanized forms of originally non-human antibodies. Even humanized antibodies can provoke the synthesis of anti-therapeutic antibodies such as those observed against 
an anti-CD25 [39] that may limit their long-term effectiveness in humans. Herceptin, used to treat certain breast cancers, carries FDA warnings for cardiomyopathy and embryo-fetal toxicity that limit its widespread use.

Small molecule-based neuroprotective strategies include anti-excitotoxic agents [40, 41], nitric oxide and iNOS inhibitors [42], anti-oxidants [43], $\mathrm{Ca}^{2+}$ channel blockers [44], $\mathrm{Na}^{+}$channel blockers [45], $\mathrm{Na}^{+} / \mathrm{Ca}^{2+}$ exchanger inhibitors and growth/neurotrophic factors [46, 47], neural peptides [48], and components of essential oils [49]. A recent study of the MS drug FTY720 reports that this small molecule induces neurite growth, alters growth cone morphology, and promotes axon regeneration [50]. FTY720 treatment of cerebellar neurons induced components of the actin cytoskeleton, which are important for axon growth, but did not alter the levels of tubulin tyrosination or acetylation. In contrast, treatment of cortical neurons with rHIgM12 increased the level of tyrosinated tubulin while decreasing the levels of acetylated tubulin, consistent with promoting a more dynamic cytoskeleton needed for axon growth.

In conclusion, our results provide direct evidence for human antibody-mediated protection of axon integrity in a mouse model of progressive MS. To our best knowledge, rHIgM12 is the first human IgM antibody that is neuroprotective in the absence of remyelination. Combined treatment using both rHIgM 22 and rHIgM12 may result in enhanced neuroprotection and better remyelination. However, in TMEV-IDD, complete repair is likely not possible due to the persistent presence of virus. In fact, some of the best histologic repair in TMEV-IDD is driven by treatment with polyclonal human IgM [15], which contains antibodies that bind to both OLs and neurons. A cocktail of human IgMs may be functionally equivalent to this limited resource.

\section{Abbreviations \\ CNS: central nervous system; MRS: magnetic resonance spectroscopy; MS: multiple sclerosis; NAA: N-acetyl-aspartate; PPMS: primary progressive multiple sclerosis; rHIgM12: recombinant human immunoglobulin M 12; rHIgM22: recombinant human immunoglobulin M 22; RRMS: relapsing remitting multiple sclerosis; SC: spinal cord; SJL: Swiss Jim Lambert; SPMS: secondary progressive multiple sclerosis; TMEV: Theiler's murine encephalomyelitis virus.}

\section{Competing interests}

Patents for antibodies that promote remyelination and CNS repair are issued and owned by Mayo Clinic. AEW and MR have a potential conflict of interest.

\section{Authors' contributions}

$M R$ and $A E W$ designed the research; BW and AD performed the research; $B W, A D, A E W$, and MR analyzed the data; and BW, AEW, and MR wrote the paper. All authors read and approved the final manuscript.

\section{Acknowledgements}

\section{Financial support}

This work was supported by grants from the NIH (R01 GM092993, R01 NS048357, and R21 NS073684), the National Multiple Sclerosis Society (CA 1060A), the Minnesota Partnership Award for Biotechnology and Medical Genomics, Mayo
Clinic Center for Translational Science Activities (CTSA), and Mayo Clinic CTSA grant number UL1 TR000135 from the National Center for Advancing Translational Science (NCATS), a component of the National Institutes of Health $(\mathrm{NIH})$ through a High-Impact Pilot and Feasibility Award (HIPFA) and Novel Methodology Award (NMDA). Additional support was provided from Mayo Clinic Center for Multiple Sclerosis and Demyelinating Diseases (CMSDD) through a gift from Dr. and Mrs. Moon Park. We acknowledge with thanks the support from the Applebaum, Hilton, Peterson and Sanford Foundations and the McNeilus family.

\section{Author details}

'Department of Neurology, Mayo Clinic College of Medicine, Mayo Clinic, 200 First Street SW, Rochester, MN 55905, USA. ${ }^{2}$ Mayo Clinic Center for Multiple Sclerosis and Autoimmune Neurology, Mayo Clinic, 200 First Street SW, Rochester, MN 55905, USA. ${ }^{3}$ Center for Regenerative Medicine, Neuroregeneration, Mayo Clinic, 200 First Street SW, Rochester, MN 55905, USA. ${ }^{4}$ Department of Immunology, Mayo Clinic College of Medicine, Mayo Clinic, 200 1st Street SW, Rochester, MN 55905, USA.

Received: 15 February 2016 Accepted: 24 April 2016

Published online: 28 April 2016

\section{References}

1. Bieber AJ, Ure DR, Rodriguez M. Genetically dominant spinal cord repair in a murine model of chronic progressive multiple sclerosis. J Neuropathol Exp Neurol. 2005;64:46-57.

2. Mecha M, Carrillo-Salinas FJ, Mestre L, Feliu A, Guaza C. Viral models of multiple sclerosis: neurodegeneration and demyelination in mice infected with Theiler's virus. Prog Neurobiol. 2013;101-102:46-64.

3. Rodriguez M, Warrington AE, Pease LR. Human natural autoantibodies in the treatment of neurologic disease. Neurology. 2009;72:1269-76.

4. Warrington $A E$, Bieber AJ, Van Keulen V, Ciric B, Pease LR, Rodriguez M. Neuron-binding human monoclonal antibodies support central nervous system neurite extension. J Neuropathol Exp Neurol. 2004;63:461-73.

5. Wright $B R$, Warrington $A E$, Edberg DD, Rodriguez M. Cellular mechanisms of central nervous system repair by natural autoreactive monoclonal antibodies. Arch Neurol. 2009;66:1456-9.

6. Denic A, Macura SI, Warrington AE, Pirko I, Grossardt BR, Pease LR, Rodriguez M. A single dose of neuron-binding human monoclonal antibody improves spontaneous activity in a murine model of demyelination. PLoS One. 2011;6:e26001.

7. Wootla B, Denic A, Watzlawik JO, Warrington AE, Rodriguez M. A single dose of a neuron-binding human monoclonal antibody improves brainstem NAA concentrations, a biomarker for density of spinal cord axons, in a model of progressive multiple sclerosis. J Neuroinflammation. 2015;12:83.

8. Ure D, Rodriguez M. Extensive injury of descending neurons demonstrated by retrograde labeling in a virus-induced murine model of chronic inflammatory demyelination. J Neuropathol Exp Neurol. 2000;59:664-78.

9. Dal Canto MC, Lipton HL. A new model of persistent viral infection with primary demyelination. Neurol Neurocir Psiquiatr. 1977;18:455-67.

10. Xu X, Wittenberg NJ, Jordan LR, Kumar S, Watzlawik JO, Warrington AE, Oh SH, Rodriguez M. A patterned recombinant human IgM guides neurite outgrowth of CNS neurons. Scientific reports. 2013;3:2267.

11. McGavern DB, Murray PD, Rodriguez M. Quantitation of spinal cord demyelination, remyelination, atrophy, and axonal loss in a model of progressive neurologic injury. J Neurosci Res. 1999;58:492-504.

12. Howe $\mathrm{CL}$, Adelson JD, Rodriguez M. Absence of perforin expression confers axonal protection despite demyelination. Neurobiol Dis. 2007;25:354-9.

13. Wootla B, Denic A, Watzlawik JO, Warrington AE, Rodriguez M. Antibody-mediated oligodendrocyte remyelination promotes axon health in progressive demyelinating disease. Mol Neurobiol. 2015 [Epub ahead of print].

14. Bruce CC, Zhao C, Franklin RJ. Remyelination-an effective means of neuroprotection. Horm Behav. 2010;57:56-62.

15. Warrington AE, Asakura K, Bieber AJ, Ciric B, Van Keulen V, Kaveri SV, Kyle $R A$, Pease $L R$, Rodriguez M. Human monoclonal antibodies reactive to oligodendrocytes promote remyelination in a model of multiple sclerosis. Proc Natl Acad Sci U S A. 2000;97:6820-5.

16. Bieber AJ, Warrington A, Asakura K, Ciric B, Kaveri SV, Pease LR, Rodriguez M. Human antibodies accelerate the rate of remyelination following lysolecithin-induced demyelination in mice. Glia. 2002;37:241-9. 
17. Mitsunaga Y, Ciric B, Van Keulen V, Warrington AE, Paz Soldan M, Bieber AJ, Rodriguez M, Pease LR. Direct evidence that a human antibody derived from patient serum can promote myelin repair in a mouse model of chronic-progressive demyelinating disease. FASEB journal : official publication of the Federation of American Societies for Experimental Biology. 2002;16:1325-7.

18. Warrington AE, Bieber AJ, Ciric B, Pease LR, Van Keulen V, Rodriguez M. A recombinant human IgM promotes myelin repair after a single, very low dose. J Neurosci Res. 2007:85:967-76.

19. Denic A, Bieber A, Warrington A, Mishra PK, Macura S, Rodriguez M. Brainstem $1 \mathrm{H}$ nuclear magnetic resonance (NMR) spectroscopy: marker of demyelination and repair in spinal cord. Ann Neurol. 2009;66:559-64.

20. Greenberg BM, Rodriguez M, Kantarci OH, Arnold D, Wang L, Carrazana EJ, Blight AR, Button DC, Caggiano AO, Eisen A. Safety and tolerability of the remyelinating therapeutic antibody $\mathrm{rH}$ IgM22 in patients with stable multiple sclerosis. Poster No - P4.339 | ACO P5130, 67th AAN Annual Meeting | American Academy of Neurology ${ }^{\circledR}$ April 18-25. Washington, DC, USA; 2015.

21. McGavern DB, Murray PD, Rivera-Quinones C, Schmelzer JD, Low PA, Rodriguez M. Axonal loss results in spinal cord atrophy, electrophysiological abnormalities and neurological deficits following demyelination in a chronic inflammatory model of multiple sclerosis. Brain. 2000;123(Pt 3):519-31.

22. Griffiths I, Klugmann M, Anderson T, Yool D, Thomson C, Schwab MH, Schneider A, Zimmermann F, McCulloch M, Nadon N, Nave KA. Axonal swellings and degeneration in mice lacking the major proteolipid of myelin. Science. 1998:280:1610-3.

23. Huizinga R, Gerritsen W, Heijmans N, Amor S. Axonal loss and gray matter pathology as a direct result of autoimmunity to neurofilaments. Neurobiol Dis. 2008;32:461-70.

24. Peterson JW, Bo L, Mork S, Chang A, Trapp BD. Transected neurites, apoptotic neurons, and reduced inflammation in cortical multiple sclerosis lesions. Ann Neurol. 2001;50:389-400.

25. Askvig JM, Watt JA. The MAPK and PI3K pathways mediate CNTF-induced neuronal survival and process outgrowth in hypothalamic organotypic cultures. Journal of cell communication and signaling. 2015;9:217-31.

26. Novikova L, Novikov L, Kellerth JO. Brain-derived neurotrophic factor reduces necrotic zone and supports neuronal survival after spinal cord hemisection in adult rats. Neurosci Lett. 1996;220:203-6.

27. Chen MS, Huber AB, van der Haar ME, Frank M, Schnell L, Spillmann AA, Christ F, Schwab ME. Nogo-A is a myelin-associated neurite outgrowth inhibitor and an antigen for monoclonal antibody IN-1. Nature. 2000;403: 434-9.

28. Bros-Facer V, Krull D, Taylor A, Dick JR, Bates SA, Cleveland MS, Prinjha RK, Greensmith $\mathrm{L}$. Treatment with an antibody directed against Nogo-A delays disease progression in the SOD1G93A mouse model of amyotrophic lateral sclerosis. Hum Mol Genet. 2014;23:4187-200.

29. Tsai SY, Markus TM, Andrews EM, Cheatwood JL, Emerick AJ, Mir AK, Schwab ME, Kartje GL. Intrathecal treatment with anti-Nogo-A antibody improves functional recovery in adult rats after stroke. Exp Brain Res. 2007; 182:261-6.

30. Zhao RR, Andrews MR, Wang D, Warren P, Gullo M, Schnell L, Schwab ME, Fawcett JW. Combination treatment with anti-Nogo-A and chondroitinase $A B C$ is more effective than single treatments at enhancing functional recovery after spinal cord injury. Eur J Neurosci. 2013;38:2946-61.

31. Placheta E, Hendry JM, Wood MD, Lafontaine CW, Liu EH, Cecilia Alvarez Veronesi M, Frey M, Gordon T, Borschel GH. The ErbB2 inhibitor Herceptin (Trastuzumab) promotes axonal outgrowth four weeks after acute nerve transection and repair. Neurosci Lett. 2014;582:81-6.

32. Xu X, Denic A, Jordan LR, Wittenberg NJ, Warrington AE, Wootla B, Papke $L M$, Zoecklein LJ, Yoo D, Shaver J. A natural human IgM that binds to gangliosides is therapeutic in murine models of amyotrophic lateral sclerosis. Dis Model Mech. 2015;8:831-42.

33. Watzlawik JO, Kahoud RJ, Ng S, Painter MM, Papke LM, Zoecklein L, Wootla B, Warrington AE, Carey WA, Rodriguez M. Polysialic acid as an antigen for monoclonal antibody HlgM12 to treat multiple sclerosis and other neurodegenerative disorders. J Neurochem. 2015;134:865-78.

34. Sheikh KA, Sun J, Liu Y, Kawai H, Crawford TO, Proia RL, Griffin JW, Schnaar RL. Mice lacking complex gangliosides develop Wallerian degeneration and myelination defects. Proc Natl Acad Sci U S A. 1999;96:7532-7.
35. Fujitani M, Kawai H, Proia RL, Kashiwagi A, Yasuda H, Yamashita T. Binding of soluble myelin-associated glycoprotein to specific gangliosides induces the association of p75NTR to lipid rafts and signal transduction. J Neurochem. 2005;94:15-21.

36. Xu X, Warrington AE, Wright BR, Bieber AJ, Van Keulen V, Pease LR, Rodriguez M. A human IgM signals axon outgrowth: coupling lipid raft to microtubules. J Neurochem. 2011;119:100-12.

37. Pepinsky RB, Shao Z, Ji B, Wang Q, Meng G, Walus L, Lee X, Hu Y, Graff C, Garber E. Exposure levels of anti-LINGO-1 Li81 antibody in the central nervous system and dose-efficacy relationships in rat spinal cord remyelination models after systemic administration. J Pharmacol Exp Ther. 2011;339:519-29.

38. Pernet $\mathrm{V}$, Schwab ME. The role of Nogo-A in axonal plasticity, regrowth and repair. Cell Tissue Res. 2012;349:97-104.

39. Tran JQ, Othman AA, Mikulskis A, Wolstencroft P, Elkins J. Pharmacokinetics of daclizumab high-yield process with repeated administration of the clinical subcutaneous regimen in patients with relapsing-remitting multiple sclerosis. Clinical pharmacology : advances and applications. 2016;8:9-13.

40. Pitt D, Werner P, Raine CS. Glutamate excitotoxicity in a model of multiple sclerosis. Nat Med. 2000;6:67-70.

41. Rosin C, Bates TE, Skaper SD. Excitatory amino acid induced oligodendrocyte cell death in vitro: receptor-dependent and -independent mechanisms. J Neurochem. 2004;90:1173-85.

42. Okuda S, Kanda F, Kawahara Y, Chihara K. Regulation of inducible nitric oxide synthase expression in L6 rat skeletal muscle cells. Am J Physiol. 1997:272:C35-40.

43. Gilgun-Sherki Y, Panet H, Holdengreber V, Mosberg-Galili R, Offen D. Axonal damage is reduced following glatiramer acetate treatment in C57/bl mice with chronic-induced experimental autoimmune encephalomyelitis. Neurosci Res. 2003;47:201-7.

44. Brand-Schieber E, Werner P. Calcium channel blockers ameliorate disease in a mouse model of multiple sclerosis. Exp Neurol. 2004;189:5-9.

45. Waxman SG. Sodium channels as molecular targets in multiple sclerosis. J Rehabil Res Dev. 2002;39:233-42.

46. Webster HD. Growth factors and myelin regeneration in multiple sclerosis. Mult Scler. 1997;3:113-20.

47. Linker RA, Maurer M, Gaupp S, Martini R, Holtmann B, Giess R, Rieckmann P, Lassmann H, Toyka KV, Sendtner M, Gold R. CNTF is a major protective factor in demyelinating CNS disease: a neurotrophic cytokine as modulator in neuroinflammation. Nat Med. 2002;8:620-4.

48. Abad C, Waschek JA. Immunomodulatory roles of VIP and PACAP in models of multiple sclerosis. Curr Pharm Des. 2011;17:1025-35.

49. Park H, Seol GH, Ryu S, Choi IY. Neuroprotective effects of (-)-linalool against oxygen-glucose deprivation-induced neuronal injury. Arch Pharm Res. 2016;39(4):555-64.

50. Anastasiadou S, Knoll B. The multiple sclerosis drug fingolimod (FTY720) stimulates neuronal gene expression, axonal growth and regeneration. Exp Neurol. 2016:279:243-60.

\section{Submit your next manuscript to BioMed Central and we will help you at every step:}

- We accept pre-submission inquiries

- Our selector tool helps you to find the most relevant journal

- We provide round the clock customer support

- Convenient online submission

- Thorough peer review

- Inclusion in PubMed and all major indexing services

- Maximum visibility for your research

Submit your manuscript at www.biomedcentral.com/submit 